\title{
Increased frequency of intentional weight loss associated with reduced mortality: a prospective cohort analysis
}

\author{
Erik A. Willis ${ }^{1,2^{*}}$ (D) Wen-Yi Huang ${ }^{1}$, Pedro F. Saint-Maurice ${ }^{1}$, Michael F. Leitzmann ${ }^{3}$, Elizabeth A. Salerno ${ }^{1}$,
} Charles E. Matthews ${ }^{1+}$ and Sonja I. Berndt ${ }^{1+}$

\begin{abstract}
Background: Due to the high prevalence of obesity and the difficulty in maintaining weight loss, repeated bouts of weight loss are a common occurrence. However, there are inconsistencies in epidemiological studies regarding repetitive weight fluctuations being associated with increased risk of mortality. Therefore, the purpose of this prospective cohort analysis was to determine the long-term association of the frequency of weight loss attempts on mortality.
\end{abstract}

Methods: This prospective cohort study used data collected from adult AARP members living in 6 states (California, Florida, Louisiana, New Jersey, North Carolina, or Pennsylvania) or 2 metropolitan areas (Atlanta, Georgia, or Detroit, Michigan) and participating in the National Institutes of Health-AARP Diet and Health Study between 2004 and 2006. Self-reported data were analyzed for 161,738 middle-aged adults. During an average 7 years of follow-up, 21 , 194 deaths were recorded. Hazard ratios of all-cause, cardiovascular, and cancer mortality were estimated adjusting for demographic, lifestyle, and behavioral risk factors.

Results: Increased frequency of weight loss attempts of at least five pounds was associated with lower mortality ( $\left.p_{\text {trend }}<0.010\right)$. Multivariate hazard ratios (95\% confidence intervals) for all-cause death among individuals who successfully attempted weight loss compared with those who did not make any attempts were $0.94(0.90-0.98)$ for $1-2$ attempts, $0.96(0.91-1.01)$ for 3-4 attempts, 0.91 (0.85-0.96) for 5-6 attempts, 0.91 (0.85-0.98) for 7-8 attempts, $0.87(0.80-0.95)$ for $9-10$ attempts, and $0.88(0.82-0.94)$ for $11+$ attempts. Similar results were noted for men and women, participants with healthy weight and overweight/obesity, and even among those who gained weight over time. Protective associations were also observed for deaths due to cardiovascular disease and cancer.

Conclusions: Increased frequency of intentionally losing at least five pounds in mid-life was associated with a lower risk of future death. Repeated attempts with moderate amounts of weight loss may provide benefit in terms of longevity.

(Continued on next page)

\footnotetext{
* Correspondence: erik.willis@unc.edu

${ }^{\dagger}$ Charles E. Matthews and Sonja I. Berndt contributed equally to this work

'Division of Cancer Epidemiology and Genetics, National Cancer Institute,

National Institutes of Health, Department Health and Human Services, Bethesda, MD, USA

${ }^{2}$ Center for Health Promotion Disease Prevention, University of North Carolina at Chapel Hill, Chapel Hill, NC, USA

Full list of author information is available at the end of the article
}

C C The Author(s). 2020 Open Access This article is licensed under a Creative Commons Attribution 4.0 International License, which permits use, sharing, adaptation, distribution and reproduction in any medium or format, as long as you give appropriate credit to the original author(s) and the source, provide a link to the Creative Commons licence, and indicate if changes were made. The images or other third party material in this article are included in the article's Creative Commons licence, unless indicated otherwise in a credit line to the material. If material is not included in the article's Creative Commons licence and your intended use is not permitted by statutory regulation or exceeds the permitted use, you will need to obtain permission directly from the copyright holder. To view a copy of this licence, visit http://creativecommons.org/licenses/by/4.0/. The Creative Commons Public Domain Dedication waiver (http://creativecommons.org/publicdomain/zero/1.0/) applies to the data made available in this article, unless otherwise stated in a credit line to the data. 
(Continued from previous page)

Trial registration number: ClinicalTrials.gov number, NCT00340015

Keywords: Obesity, Weight loss, Mortality, Prospective cohort

\section{Introduction}

With the rise in obesity prevalence worldwide and its negative impact on health [1-4], weight management has become a key public health focus. Studies have consistently shown health benefits associated with clinically significant weight loss (3-5\%) [5-8], and it is recommended that individuals who are classified as overweight/obese lose weight gradually ( 1-2 lbs/week) [9] using evidence-based approaches [10]. The proportion of US adults who report intentionally losing weight is substantial and has increased from $43 \%$ in 2007 to $49 \%$ in 2016 [11]. However, approximately $65-80 \%$ of individuals who intentionally achieve clinically meaningful weight loss will regain some, if not all, of the weight lost within 12 months after stopping treatment [12, 13]. As a consequence, repeated bouts of intentional weight loss is a common occurrence [14], with some reporting as many as 50 weight loss attempts over their lifetime [15],

Early epidemiological studies suggested that repetitive weight fluctuations are associated with increased risk of mortality [16-20], several chronic diseases [21], and poor mental health [22], which called into question the benefits of recommending adults with overweight/obesity to lose weight [23]. However, recent evidence suggests there is no association between repeated bouts of weight loss and negative health outcomes, body composition, or future obesity [24, 25]. Such inconsistencies may be due to lack of information on total volume of weight lost and/or type of weight loss (e.g., intentional versus unintentional) or failure to control for important confounders (e.g., smoking, body mass index [BMI], overall weight trajectories). It is particularly important to differentiate unintentional from intentional weight loss, since weight loss due to illness may be reflective of poor health [26], which could confound the association. To clarify these issues, we evaluated the association of frequency of intentionally losing at least five pounds with all-cause and cause-specific mortality in middle-aged adults in the National Institutes of Health (NIH)-AARP Diet and Health Study (ClinicalTrials.gov number, NCT00340015).

\section{Methods}

\section{Study population}

As described previously [27], the NIH-AARP Study is a prospective cohort of 566,407 men and women aged 5071 years who returned a baseline questionnaire (BQ) eliciting information on demographic and health-related behaviors between 1995 and 1996. A second questionnaire
(Risk Factor Questionnaire; RFQ), which collected more detailed information about anthropometric characteristics at different ages, was sent subsequent to the BQ in late 1996 to respondents still living in the study area and not having prevalent cancer of the prostate, breast, or colon. A third questionnaire (Follow-up Questionnaire; FQ) asking detailed questions about lifestyle behaviors, including intentional weight loss, was completed by 313,363 participants in 2004-2006. The NIH-AARP Study was approved by the Special Studies Institutional Review Board of the National Cancer Institute, and all participants gave written informed consent by completing and returning the questionnaire.

Of the 419,154 non-proxy respondents who returned either the RFQ or FQ, we excluded those without FQ data ( $n=124,284)$. While exact reasons for not completing FQ are unknown, from death record ascertainment, we are able to determine only $21 \%$ of those excluded died prior to FQ and $81 \%$ had self-reported their health condition at time of RFQ as Good-Excellent. Furthermore, we excluded those with extreme frequency and volume of weight loss (twice the interquartile range; $n=1315$ ) and those that were underweight or with extreme BMI values $\left(<18.5 \mathrm{~kg} / \mathrm{m}^{2}\right.$ or $>60.0 \mathrm{~kg} / \mathrm{m}^{2}$ on the RFQ or FQ) or $\mathrm{did}$ not have BMI for at least two time points $(n=24,799)$. Finally, we excluded those with missing information on intentional weight loss $(n=107,018)$. The analytic cohort included 161,738 participants $(100,416$ men and 61,322 women). The socio-demographics and behavioral characteristics of this analytic cohort were broadly similar to all potentially eligible participants (data not shown).

\section{Assessment of weight loss frequency}

The frequency of losing at least five pounds in the past 20 years was assessed by self-report on the FQ (Additional file 1: Fig. S1), by summing the total number of times participants reported losing weight using the mid-point value in each frequency category. Participants were then categorized by frequency of weight loss (never, 1-2, 3-4, 5-6, 7-8, 9-10, and $11+$ attempts). The average volume (i.e., lbs) of weight loss per attempt was calculated by dividing total volume of weight loss (based on summing the mid-point values for loss) over all attempts by the total frequency of attempts.

\section{Assessment of historical weight, BMI, and other covariates}

Participants reported their height and current weight on both the BQ and FQ, and historical weights at age 35 and 
50 on the RFQ, from which we calculated age-specific BMIs $\left(\mathrm{kg} / \mathrm{m}^{2}\right)$. To determine the participant's starting BMI that preceded the 20-year intentional weight loss period that ended at FQ, we used the "historical" BMI at either age 35 or 50, whichever was closest to the participant's age at the FQ minus 20 years. Historical BMI was categorized as healthy weight $\left(18.5-<25.0 \mathrm{~kg} / \mathrm{m}^{2}\right)$, overweight $\left(25.0-<30.0 \mathrm{~kg} / \mathrm{m}^{2}\right)$, obese $\left(30.0-<35.0 \mathrm{~kg} / \mathrm{m}^{2}\right)$, and severely obese $\left(\geq 35.0 \mathrm{~kg} / \mathrm{m}^{2}\right)$. Other covariates, such as physical activity and sedentary time, were based on self-reported data collected on the FQ.

\section{Death ascertainment}

Mortality was compiled via the National Death Index, through December 2011. Causes of death were classified using the International Classification of Diseases codes. End points for our analysis were all-cause-, cancer(ICD: 140-208, 238.6, C00-C97), and cardiovascularmortality (ICD: 390-398, 401-404, 410-429, 440-448, I00-I13 I20-I51, I60-I78). Vital status ascertainment in this cohort was $>95 \%[28]$.

\section{Statistical analysis}

Individual participant linear slopes for BMI were derived using linear mixed models with fixed and random effect for time. Models were run by sex to account for sexspecific differences in weight across time. Participants were then classified as weight losers (slope $<25$ th percentile), weight maintainers (slope 25 th to 75 th percentile), and weight gainers (slope $\geq 75$ th percentile). Additionally, due to weight change of as little as 3\% being associated with changes in health outcomes [5-8], sensitivity analysis of participants categorized as weight gainers ( $>+3 \%$ BMI change), maintainers $(-3 \%$ to $+3 \%)$, or losers $(<-3 \%)$ were completed. Results of analyses that included weight change as $\pm 3 \%$ did not differ meaningfully; therefore, models with weight change categorized by linear slopes are reported (see Additional file 1: Table S6).

Cox proportional hazard models, with age as the underlying time metric, were fit to estimate hazard ratios (HRs) and 95\% confidence intervals (CIs) for mortality across increasing frequency of successful weight loss attempts. Participants were followed prospectively from the date of the FQ completion (i.e., on which individuals reported previous weight loss attempts) to either death or end of follow-up (December 31, 2011), whichever came first.

We assessed mortality risk in two models: (1) adjusting for age, sex, race/ethnicity, education level, healthy eating index, physical activity, sedentary time, smoking, number of chronic diseases, overall health, marital status, age at retirement, age at menopause (for women) and historical BMI and (2) additionally adjusting for weight change categories (i.e., gainer, maintainer, loser). Tests of linear trends were conducted by modeling the median value from each frequency of weight loss attempt category as a continuous variable, after exclusion of the unintentional weight loss group. In secondary analyses, we stratified by median age at start of intentional weight loss period $(<51, \geq 51$ years), sex, historical BMI $\left(\geq 18.5-<25.0 \mathrm{~kg} / \mathrm{m}^{2}, \geq 25.0 \mathrm{~kg} / \mathrm{m}^{2}\right)$, weight change categories, and smoking status (never, former, current). Missing data for covariates were treated as a separate category and included in the models. Because competing risks may play a role in estimating causespecific mortality, sub-distribution hazard models were evaluated for cancer and CVD specific mortality [29].

As a sensitivity analysis, to evaluate the possible impact of excluding participants with missing data on intentional weight loss, we used the multiple imputation method in which we regressed these measures on a number of other individual-level variables, including age, sex, race/ethnicity, education level, healthy eating index, physical activity, sedentary time, smoking, chronic diseases, overall health, marital status, age at retirement, age at menopause (for women), historical BMI, and weight change [30]. Imputed estimates and variance from 10 imputed datasets were combined to obtain the final estimated $\mathrm{HRs}$ and 95\% confidence intervals (CIs).

To evaluate associations with total weight lost volume over 20 years and frequency of successful weight loss attempts, we jointly classified frequency and total volume of weight loss categories (5-50 lbs, 50-100 lbs, 100-150 $\mathrm{lb}$., and $150+\mathrm{lbs})$. All analyses were conducted using SAS version 9.4 (SAS Institute Inc., Cary, NC).

\section{Results}

In the analytic cohort $(N=161,738)$, mean age at the start of the weight loss observation period was 51 years (range, 39-63 years). Approximately 62\% of participants were male, $93 \%$ non-Hispanic white, and 6\% current smokers. Of those reporting intentional weight loss, the median number of attempts of at least five pounds over the past 20 years was 4 (range 2-23) with an average of $\sim 11$ pounds lost per attempt. Participants with more frequent attempts were more likely to be female, former/ current smokers, overweight/obese at the beginning of the weight loss period, have $5+$ medical conditions and self-report their health as fair/poor compared those with no or few attempts (Table 1); however, there were no substantial differences in levels of physical activity, sedentary behavior, or diet quality (Table 1). Across the median 7.1 years of follow-up, 21,194 deaths occurred.

\section{Frequency of weight loss and all-cause mortality}

In multivariate models increasing frequency of weight loss of five pounds or more was associated with lower 


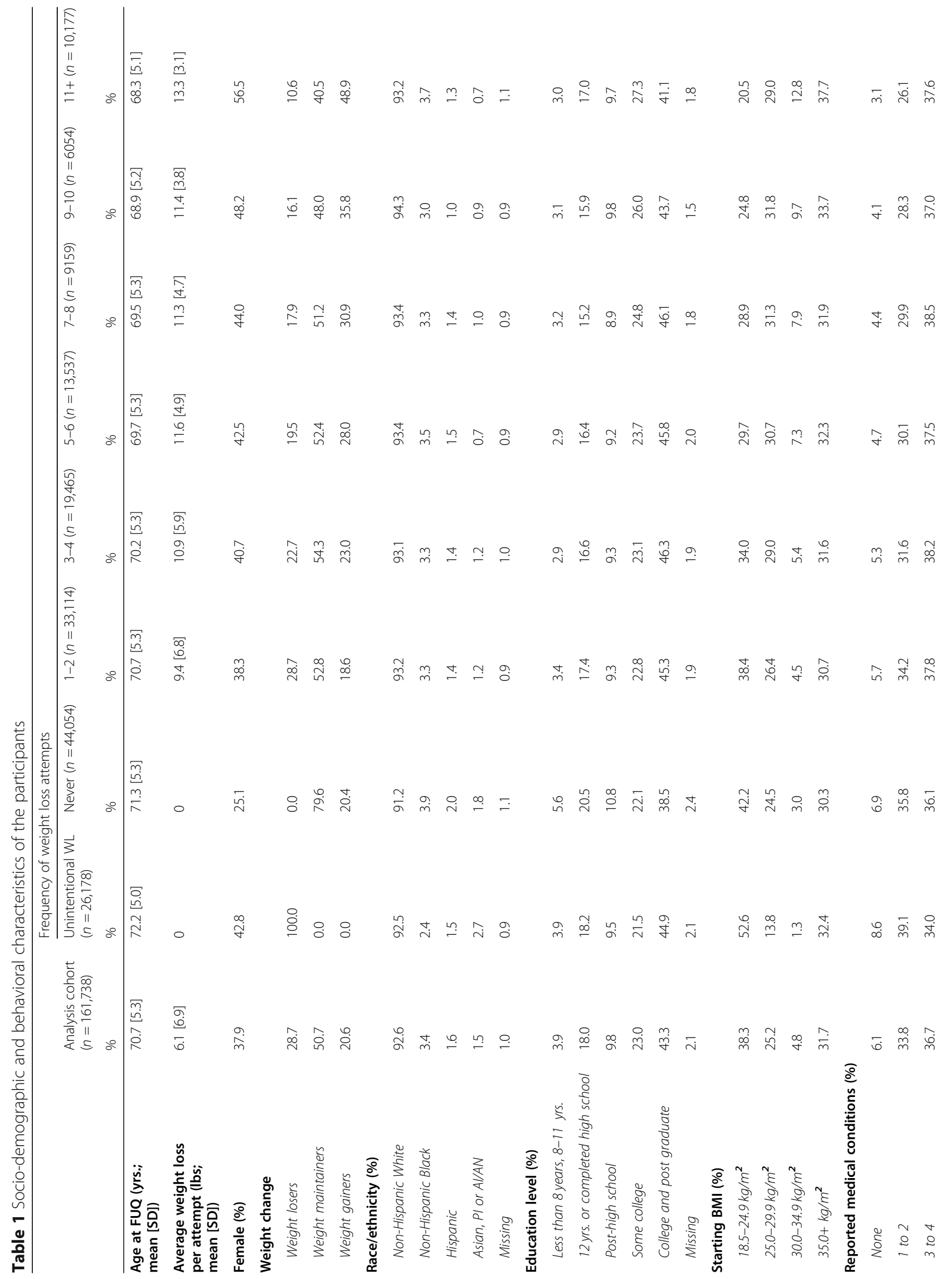




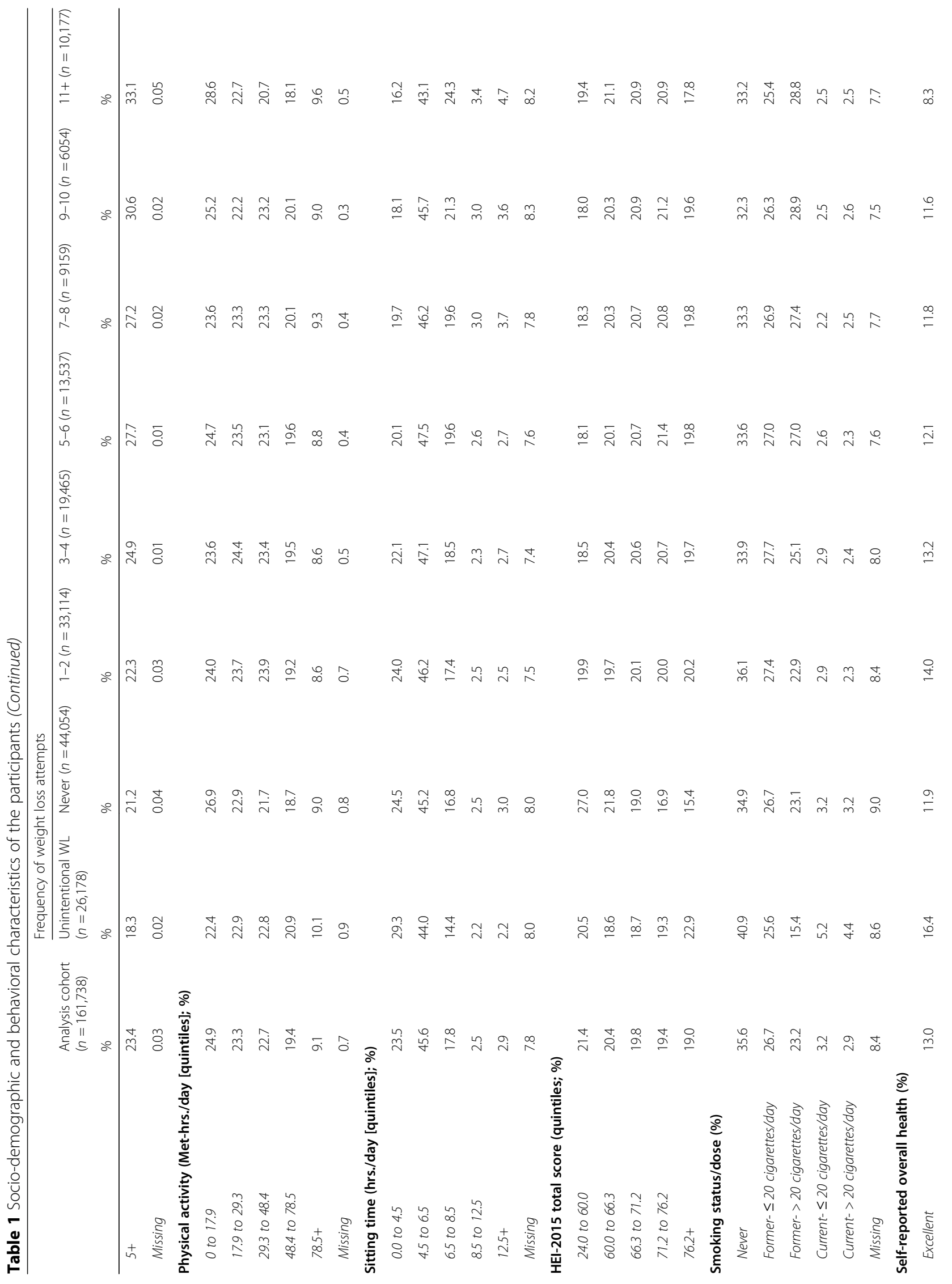




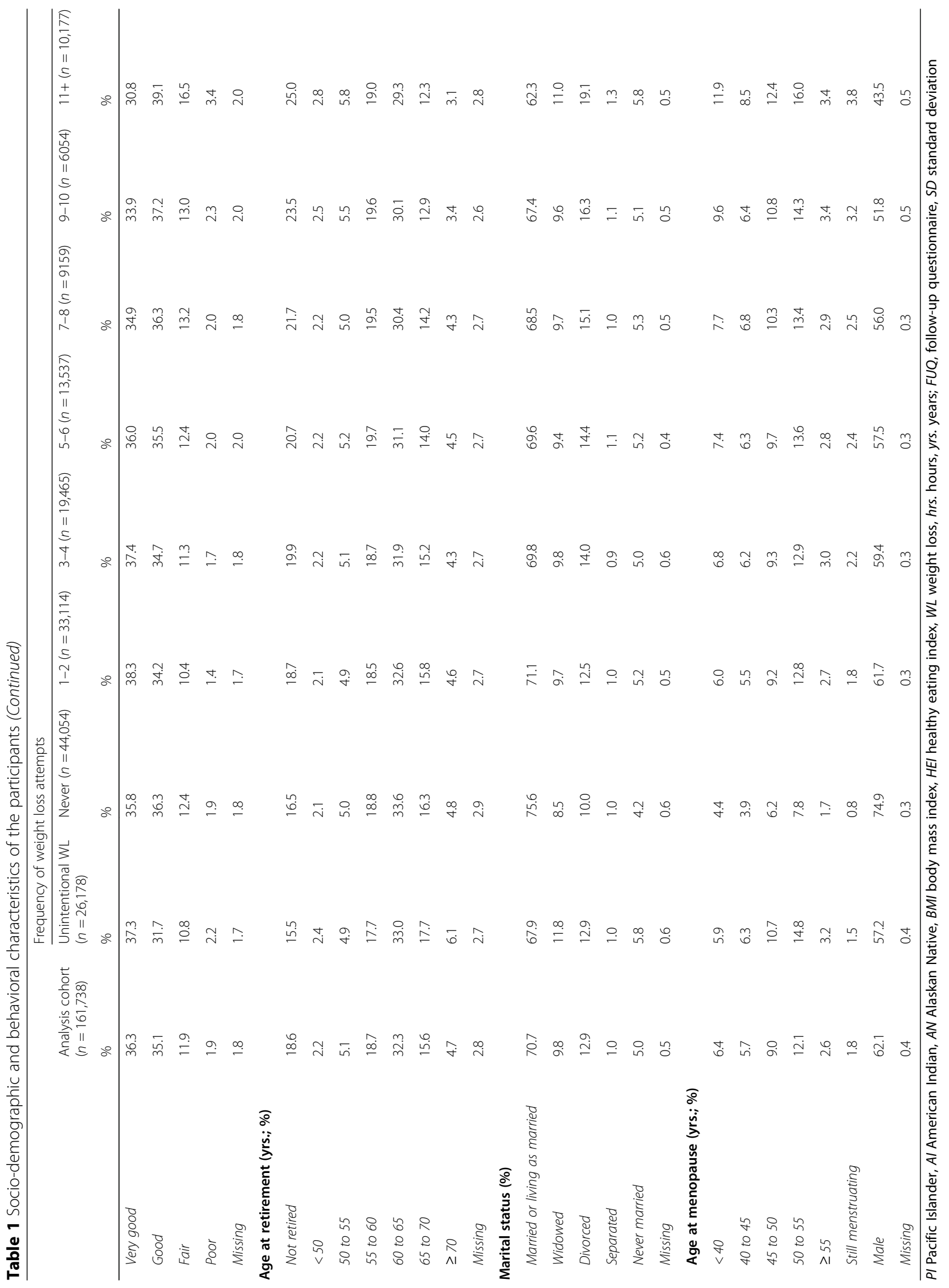


all-cause mortality ( $p$ trend $<0.0001$; Fig. 1 ; Additional file 1: Table S1). As compared to participants who never attempted weight loss, the highest frequency of weight loss attempts $(11+)$ was associated with a 9\% (95\% CI, 3-15\%) lower risk of all-cause mortality. When the model was further adjusted for weight change over the 20-year period, the associations remained ( $p$ trend $=$ $0.010)$ and were stronger across all categories of successful weight loss attempts (e.g., $\mathrm{HR}=0.88,95 \% \mathrm{CI}=0.82-$ 0. 94 for $11+$ attempts).

Those who reported no intentional weight loss attempts but lost weight over the 20 -year period (i.e., unintentional weight loss) had higher mortality risk (HR = $1.38,95 \% \mathrm{CI}=1.33-1.44)$ compared to participants who never attempted weight loss but maintained or gained weight. This association was no longer associated after adjustment for weight change categories $(\mathrm{HR}=1.01,95 \%$ $\mathrm{CI}=0.95-1.07$ ).

The inverse association between weight loss attempts and total mortality was similar when stratified by age (Additional file 1: Table S2), for men and women (Additional file 1: Table S1), and participants who were overweight/obese or had healthy BMI at the beginning of the weight loss period (Fig. 2 (A); Additional file 1: Table
S2). Even among those who gained weight over the time period, an inverse association was observed with 19\% (95\% CI, 10-27\%) lower risk of mortality for those with 11+ attempts (Fig. 2 (B) and Additional file 1: Table S2). There were no associations observed for weight maintainers (HR $=0.94,95 \%$ CI $0.84-1.04)$ or weight losers $(\mathrm{HR}=1.03,95 \% \mathrm{CI} 0.88-1.21)$ with $11+$ weight loss attempts. Across smoking status strata, an inverse association was observed between weight loss attempts and total mortality among never and former smokers, but not current smokers (Additional file 1: Table S2). Results were similar when we used multiple imputation to evaluate that possible impact of excluding participants with missing values on intentional weight loss (Additional file 1: Table S5).

To explore whether the observed associations were driven by total volume of weight lost, we jointly classified categories of total weight lost over the 20 years and frequency of those attempts and estimated the average amount of weight loss per attempt. (Fig. 3 and Additional file 1: Table S3). Within most categories of total weight loss, there was a trend toward reduced mortality with increased frequency of attempts. However, there was some evidence that the association between

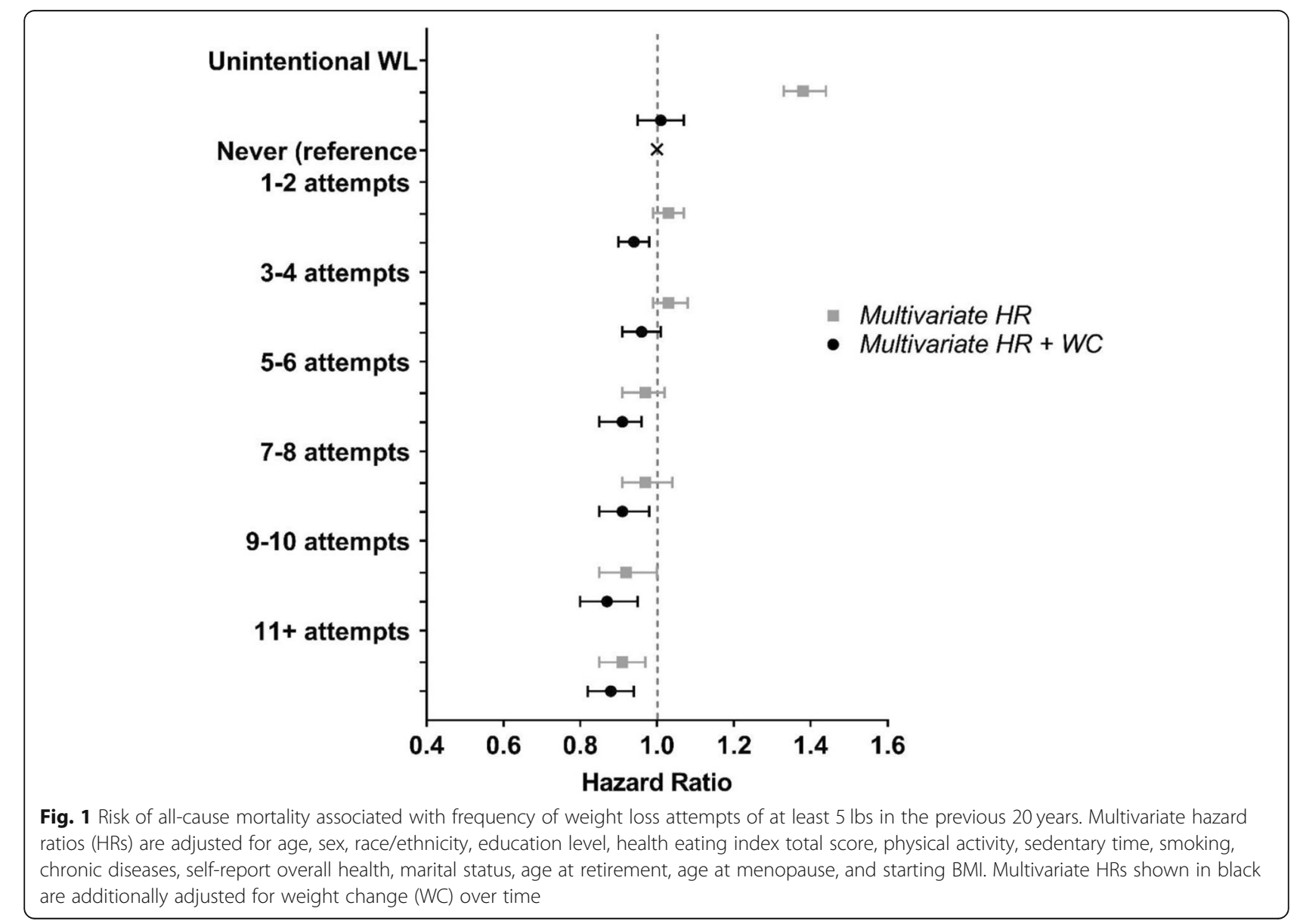




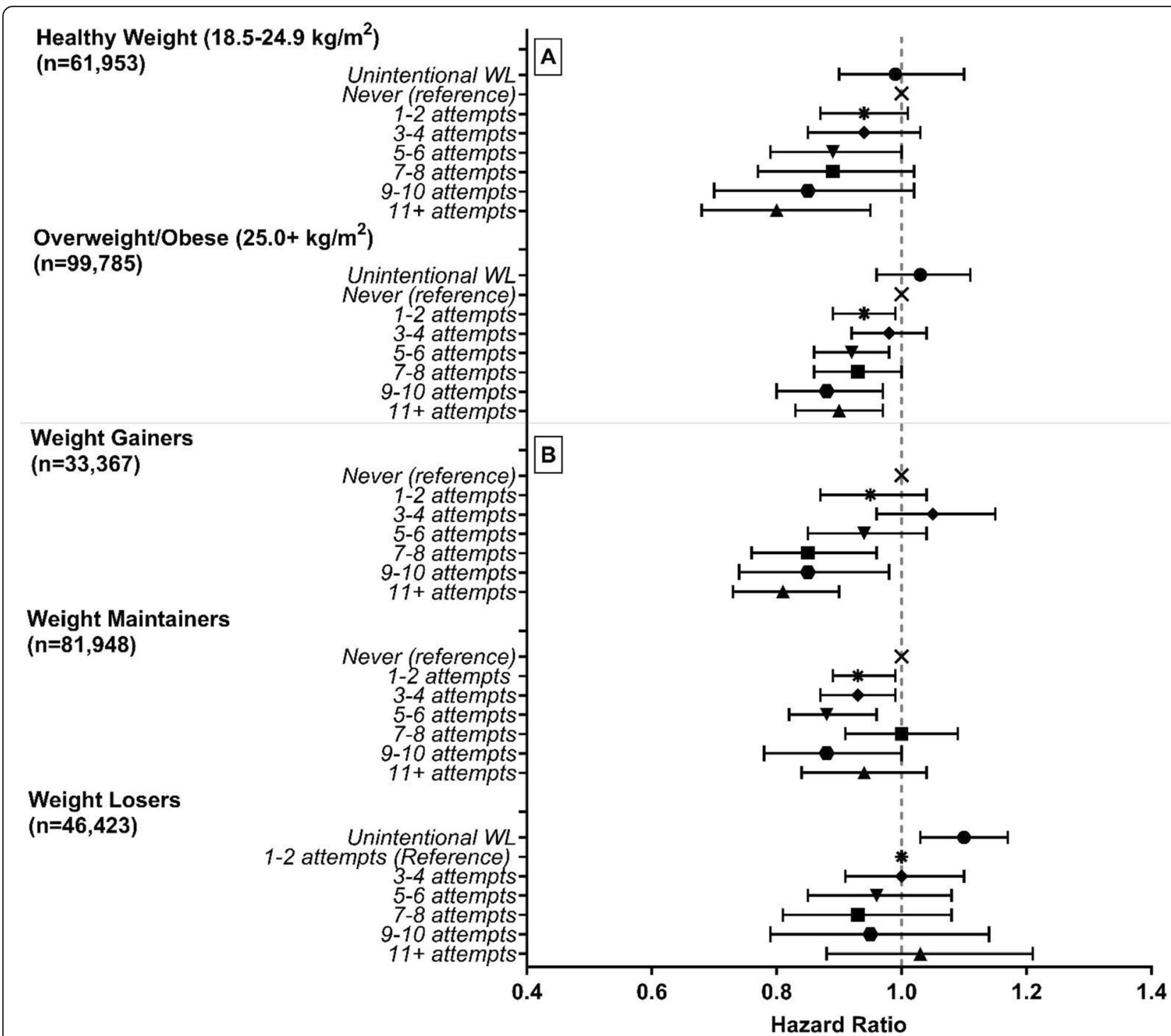

Fig. 2 Stratified analysis of proportional hazard ratios for all-cause mortality associated with frequency of weight loss (WL) attempts of at least 5 Ibs over the previous 20 years, by (A) historical BMI and (B) life course weight loss. Hazard ratios and 95\% confidence intervals (CI) are adjusted for age, sex, race/ethnicity, education level, healthy eating index total score, physical activity, sedentary time, smoking, chronic diseases, selfreported overall health, marital status, age at retirement, age at menopause, starting BMI, and weight change. Weight losers: reference group $=1-2$ attempts

frequency of weight loss and mortality was modified by total weight loss volume. For example, reduced risk was observed in those with 5-6 weight loss attempts who lost $5-50 \mathrm{lbs}(\mathrm{HR}=0.84 ; 95 \% \mathrm{CI}=0.78-0.90)$, but no association in those who lost $100-150 \mathrm{lbs}$ with the same number of attempts ( $\mathrm{HR}=0.95 ; 95 \% \mathrm{CI}=0.76-1.19)$. For large amounts of total weight loss (e.g., 100-150 lbs), more frequent attempts $(9+)$ with more moderate losses per attempt (12 lbs/attempt) were associated with lower risk (HR $=0.91,95 \%$ CI 0.84-0.98), while fewer attempts with larger losses per attempt (30 lbs/attempt) were not $(\mathrm{HR}=1.19,95 \%$ CI 0.98-1.46).

\section{Frequency of weight loss and cause-specific mortality}

The dose-response pattern of association with increasing frequency of weight loss attempts was apparent for both cancer mortality (7850 deaths) and cardiovascular mortality (6430 deaths, Fig. 4 and Table Additional file 1: S4). For cancer mortality, there was an inverse association with increasing frequency of weight loss attempts with the highest frequency of weight loss attempts $(11+)$ having a $22 \%(95 \% \mathrm{CI}, 12-30 \%)$ lower risk. For cardiovascular mortality, there was no significant association observed for any frequency group. However, a significant linear trend for both cancer $(p=0.004)$ and CVD 


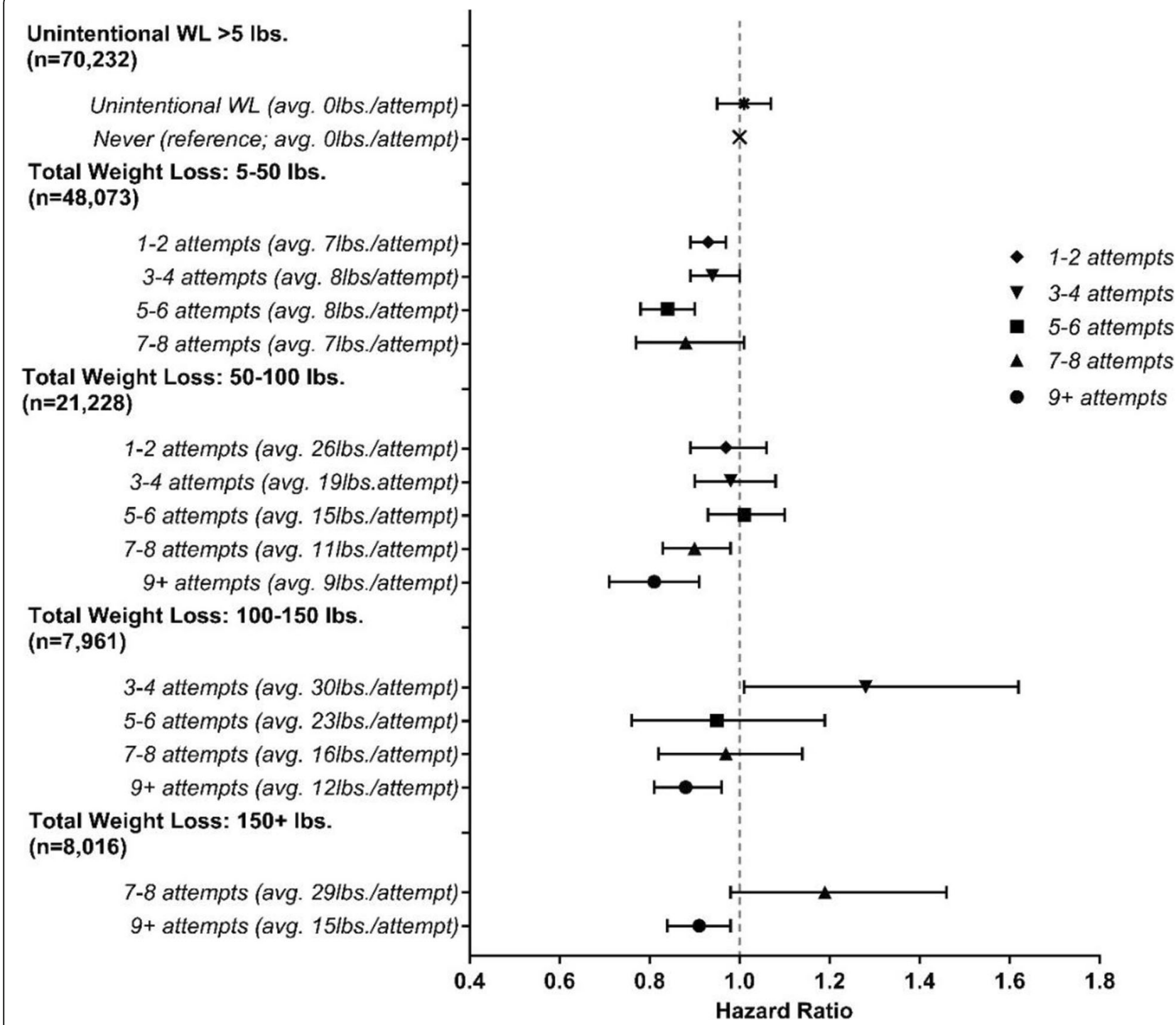

Fig. 3 Proportional hazard ratios for all-cause mortality based on the joint effects of the frequency of weight loss attempts and the total weight loss based on the sum of those attempts the previous 20 years $(n=161,738)$. Hazard ratios and 95\% confidence intervals $(\mathrm{Cl})$ adjusted for age, sex, race/ethnicity, education level, healthy eating index total score, physical activity, sedentary time, smoking, chronic diseases, self-report overall health, marital status, age at retirement, age at menopause, starting BMI, and weight change

mortality ( $p=0.021$; Additional file 1 : Table S4) was observed. After additionally controlling for weight change, the linear trends approached significance (both $p<$ 0.065; Additional file 1: Table S4).

\section{Discussion}

In this large prospective US cohort, we observed that increased frequency of weight loss attempts of five pounds or more in middle-aged adults was associated with lower future mortality risk. As compared with individuals who never intentionally lost at least five pounds, individuals who had $11+$ attempts over 20 years had a $12 \%$ lower risk of death. Notably, this inverse association was observed even among those who gained weight over the 20-year period, suggesting some benefit to frequent attempts at weight loss even if weight is eventually gained over time. Inverse associations were also evident in men and women and participants who were initially healthy weight or overweight/obese. Associations were also noted for deaths caused by cardiovascular disease and cancer.

Our findings are consistent with a recent animal study by Smith and colleagues [31], who randomized obese mice to ad libitum feeding to sustain obesity, calorie restriction to achieve a "normal" or intermediate body weight, or weight cycling (repeated episodes of calorie restriction and ad libitum refeeding). As compared to the sustained obesity group, mice who had periodic 


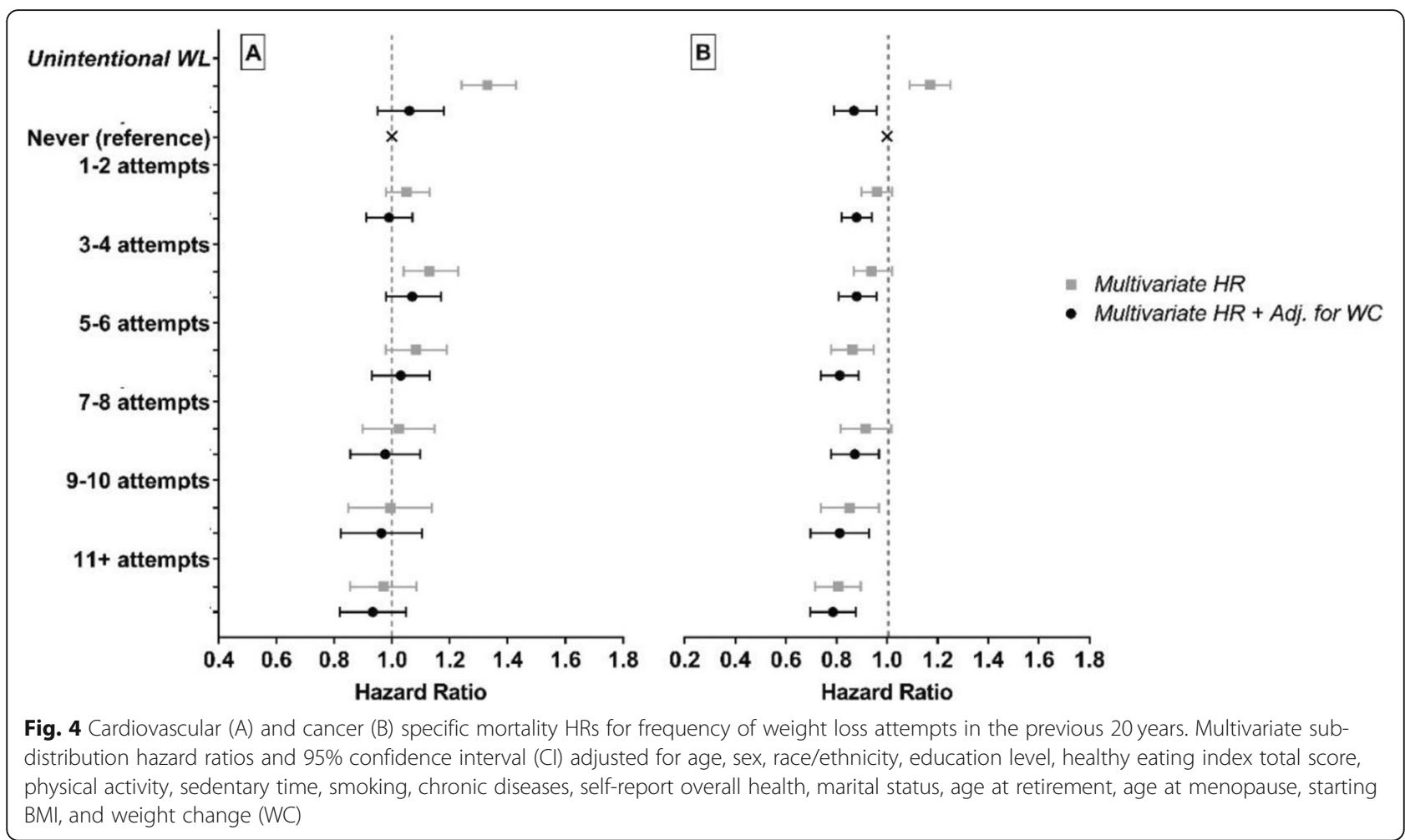

weight losses showed $43 \%$ lower mortality risk. Furthermore, recent studies suggest that periodic fasting cycles play a beneficial role by promoting the activation of degradation and turnover pathways that promote repair and removal of damaged macromolecules in humans [32-35]. Combined with our results, this evidence suggests that middle-aged adults may benefit from repeated weight loss attempts in terms of longevity, even if overall individuals gain weight over time.

Previous studies assessing weight cycling over time and mortality risk have yielded mixed results [16-20, 36, 37]. Studies evaluating weight change based on measured or self-reported weights at discrete time intervals (e.g., biennial visits) have largely observed an increased risk of mortality with fluctuations in weight; however, these studies did not differentiate between intentional and unintentional weight loss and did not evaluate weight fluctuations, including weight loss attempts, between visits or questionnaires. The increased mortality observed in these studies may be more reflective of individuals with episodes of significant unintentional weight loss due to illness and/or those engaging in more extreme dieting. Although the numbers were small in our study, we did not see a mortality benefit for those with large amounts of weight lost (e.g., 100+ lbs) over few attempts, and those with unintentional weight loss were at increased risk of mortality. In addition, variability in covariates adjusted for in previous studies of weight change may also explain differences between these studies and ours. For example, not all studies adjusted for baseline BMI, and others did not adjust for education or other potential confounders. The importance of adjusting for BMI was highlighted by the results by Stevens et al. [37], in which weight cycling appeared harmful in models not adjusted for BMI, but not in models accounting for BMI. We adjusted for a large number of important confounding factors, including BMI, physical activity, and sedentary time, in our analysis, and this may have helped clarify an independent association not seen in previous studies.

In contrast to studies of weight change based on fixed time points, studies evaluating weight cycling based on self-reported intentional weight loss attempts have not observed an increased risk of mortality. Consistent with our study, Field et al. [36] reported a reduced risk of mortality for weight cyclers based on weight loss attempts over 20 years. Similarly, examining only those who purposefully lost and regained 10+ pounds, Stevens et al. [37] found lower mortality for those lost and gained weight 1-4 or 5-9 times, although no association with those who lost and regained weight 20 or more times over their lifetime. Unlike the study by Stevens et al. [37], we did not have data on weight regained after each attempt and so could not examine the number of times that individuals regained the weight lost. While nearly all individuals regain weight previously lost, some do not and those that do may do so at different rates [38]. By adjusting for weight change over the time, we were able to account for the cumulative effects of weight loss and gain in our analysis, even if not the individual effects. 
A unique aspect of our analysis is the joint effects models of total volume of weight lost and frequency of attempts. Infrequent attempts with large volumes of weight lost provided no mortality benefit while more frequent attempts with moderate amounts of weight loss per attempt was associated with lower mortality risk. This suggests that repeated moderate amount of weight loss may provide more long-term benefit than losing a large amount of weight all at once. This is consistent with the recommendation that individuals should lose weight gradually (1-2 lbs/week) [9], and the benefit observed for frequent weight loss attempts may be because these individuals engage in healthy behaviors (e.g., exercise, healthy diet, etc.) over a cumulative longer time period than those who made no attempts. The increased risk observed with large losses of weight with infrequent attempts may be explained by possible unhealthy behaviors (e.g., meal skipping, diet pills, laxatives, diuretics, or purging) [39] that individuals may engage in to lose high volumes of weight in a single weight loss attempt. Thus, this study presents a novel analysis addressing the joint effects of total volume of weight lost and frequency of attempts not previously studied. Also, our study differed from previous studies [16-20] that did not differentiate between intentional and unintentional weight loss, investigations [16-20] that failed to adjust for BMI at baseline, and studies $[16-20,36]$ that did not adjust for other potential confounding variables.

The association between frequency of weight loss attempts and mortality was strongest among individuals who gained weight over time with limited or no benefit for those that lost or maintained their weight. We hypothesize that overall loss or maintenance of weight is the primary driver of longevity. Among those who achieve weight loss or maintenance, the frequency of the number of attempts may be less important because they have been successful in overall reducing or maintaining their weight and thus have gained the benefit in longevity. The frequency of weight loss attempts may be more important for those who ultimately gain weight, as they are at higher risk and do not have the benefit of achieving long-term weight loss or maintenance. The frequency of attempts may be reflective of increased attempts at engaging in healthy behaviors, leading to reduced mortality seen in those ultimately gaining weight.

One potential limitation of our study is that associations could reflect confounding by unmeasured or poorly measured confounders, including other unmeasured behavior changes. Although we cannot rule out residual confounding, the availability of detailed data enabled us to comprehensively adjust for many possible confounders, including BMI and sedentary time. Since past frequency of weight loss attempts was self-reported, some measurement error is inevitable due to inaccurate recall, but this misclassification is likely non-differential and presumably only biased our results toward the null. The subjects were prospectively followed for mortality after completing the questionnaire, so it is unlikely there were any systematic differences in recall between those who later died and those who did not. Second, information on previous weight loss attempts was not available for all participants. Although it is possible that participants missing weight loss information are different than the rest of the cohort, we found that those who were excluded due to missing data were broadly similar in socio-demographics and behavioral characteristics to those who were included, and no differences in mortality were observed based on death records. The results were similar after imputation to account for missing data, suggesting that our findings are valid. Third, we lack data on how and when weight loss was attempted over the 20-year period, thus, we were unable to examine the influence on mortality by weight loss methods (e.g., diet, physical activity, pharmacotherapy, bariatric surgery), timing of weight loss methods, duration of each attempt, or the weight loss attempts over shorter periods of time (e.g., < 20 years). The follow-up time was relatively short for mortality in our study (e.g., 7 years on average), but we had a large number of deaths $(N=21,194)$ giving us substantial power to detect an association. Future studies may benefit from longer follow-up. Finally, the generalizability of our results may be limited because our cohort was primarily composed of non-Hispanic Caucasian middle-aged adults that were highly educated. Therefore, future research is encouraged to investigate these associations in more diverse study populations.

Our prospective study with its large sample is the first to evaluate the long-term effects of both the frequency of intentional weight loss attempts over time and the joint effects of frequency with total weight loss. The protective association observed with increasing frequency of weight loss attempts suggests benefit even for those who have difficulty maintaining weight loss. There are plausible mechanisms by which intentional weight loss may provide longevity benefits. In addition to the increased time living with lower levels of fat mass, healthy weight loss attempts are typically accompanied by changes in other healthy behaviors, specifically better eating behaviors, reduced alcohol consumption, and increased physical activity [10]. We did not observe more healthy behaviors in those with frequent weight loss attempts based on self-reported data, but those changes may have only occurred during the period of a weight loss attempt and therefore may have not been captured by the questionnaire. Regardless if the healthy behaviors were maintained, individuals with more frequent intentional weight loss attempts may have had more total time exposed to these healthy behaviors and less years living 
with overweight/obesity over a lifetime compared to those never successfully attempting weight loss. In combination, weight loss attempts and these other health behaviors may be important for longevity.

\section{Conclusion}

In this large prospective cohort study, we discovered that more frequent intentional weight loss attempts over a 20 -year period in mid-life was associated with a reduced risk of death, even among those who ultimately gained weight. The benefits were more evident among those who lost moderate amounts of weight frequently as opposed to those who underwent a few very large weight losses. If replicated, this finding is of high clinical importance due to the increased prevalence of obesity and the difficulty in maintaining weight loss. Although repeated bouts of weight loss followed by weight regain may not be ideal, they are a common occurrence. Our results suggest that frequent intentional weight loss attempts are not harmful and may provide long-term benefit.

\section{Supplementary information}

Supplementary information accompanies this paper at https://doi.org/10. 1186/s12916-020-01716-5.

Additional File 1: Fig. S1. Assessment of frequency and volume of intentional weight loss. Table S1. All-Cause Mortality HRs for Frequency of Weight Loss Attempts; overall and by sex. Table S2. All-Cause Mortality HRs for Frequency of Weight Loss Attempts; by historical BMI, weight change, age, and smoking status. Table S3. Mortality HRs for Joint Effects of Total Weight loss and frequency of Weight Loss Attempts. Table S4. Sub-distribution cause-specific Mortality HRs for Frequency of Weight Loss Attempts. Table S5. All-Cause Mortality HRs for Frequency of Weight Loss Attempts with imputed missing data. Table S6. Comparison of Weight Change Definitions for Stratified All-Cause Mortality HRs for Frequency of Weight Loss Attempts.

\section{Acknowledgements}

This research was supported [in part] by the Intramural Research Program of the NIH, National Cancer Institute. Cancer incidence data from the Atlanta metropolitan area were collected by the Georgia Center for Cancer Statistics, Department of Epidemiology, Rollins School of Public Health, Emory University, Atlanta, Georgia. Cancer incidence data from California were collected by the California Cancer Registry, California Department of Public Health's Cancer Surveillance and Research Branch, Sacramento, California. Cancer incidence data from the Detroit metropolitan area were collected by the Michigan Cancer Surveillance Program, Community Health

Administration, Lansing, Michigan. The Florida cancer incidence data used in this report were collected by the Florida Cancer Data System (Miami, Florida) under contract with the Florida Department of Health, Tallahassee, Florida. The views expressed herein are solely those of the authors and do not necessarily reflect those of the FCDC or FDOH. Cancer incidence data from Louisiana were collected by the Louisiana Tumor Registry, Louisiana State University Health Sciences Center School of Public Health, New Orleans, Louisiana. Cancer incidence data from New Jersey were collected by the New Jersey State Cancer Registry, The Rutgers Cancer Institute of New Jersey, New Brunswick, New Jersey. Cancer incidence data from North Carolina were collected by the North Carolina Central Cancer Registry, Raleigh, North Carolina. Cancer incidence data from Pennsylvania were supplied by the Division of Health Statistics and Research, Pennsylvania Department of Health, Harrisburg, Pennsylvania. The Pennsylvania Department of Health specifically disclaims responsibility for any analyses, interpretations, or conclusions. Cancer incidence data from Arizona were collected by the Arizona Cancer Registry, Division of Public Health Services, Arizona Department of Health Services, Phoenix, Arizona. Cancer incidence data from Texas were collected by the Texas Cancer Registry, Cancer Epidemiology and Surveillance Branch, Texas Department of State Health Services, Austin, Texas. Cancer incidence data from Nevada were collected by the Nevada Central Cancer Registry, Division of Public and Behavioral Health, State of Nevada Department of Health and Human Services, Carson City, Nevada.

We are indebted to the participants in the NIH-AARP Diet and Health Study for their outstanding cooperation. We also thank Sigurd Hermansen and Kerry Grace Morrissey from Westat for study outcomes ascertainment and management and Leslie Carroll at Information Management Services for data support and analysis.

\section{Authors' contributions}

EW, CM, and SB conceptualized the study. EW organized and analyzed the data. EW, CM, SB, PS, WH, ML, and ES gave critical feedback and contributions to the manuscript. All authors read and approved the final manuscript

\section{Funding}

This work was supported by the Intramural Research Program of the National Institutes of Health National Cancer Institute.

\section{Availability of data and materials}

The datasets used and/or analyzed during the current study are available from the National Institutes of Health's National Cancer Institute.

Ethics approval and consent to participate

The NIH-AARP Study was approved by the Special Studies Institutional Review Board of the National Cancer Institute, and all participants gave written informed consent by completing and returning the questionnaire.

\section{Consent for publication}

Not applicable

\section{Competing interests}

None

\section{Author details}

'Division of Cancer Epidemiology and Genetics, National Cancer Institute, National Institutes of Health, Department Health and Human Services, Bethesda, MD, USA. ${ }^{2}$ Center for Health Promotion Disease Prevention, University of North Carolina at Chapel Hill, Chapel Hill, NC, USA. ${ }^{3}$ Department of Epidemiology and Preventive Medicine, University of Regensburg, Regensburg, Germany.

Received: 3 March 2020 Accepted: 21 July 2020

Published online: 17 September 2020

\section{References}

1. Lauby-Secretan B, Scoccianti C, Loomis D, Grosse Y, Bianchini F, Straif K. Body fatness and cancer-viewpoint of the IARC Working Group. N Engl J Med. 2016;375(8):794-8.

2. Blissmer B, Riebe D, Dye G, Ruggiero L, Greene G, Caldwell M. Health-related quality of life following a clinical weight loss intervention among overweight and obese adults: intervention and 24 month follow-up effects. Health Qual Life Out. 2006;4(1):43.

3. Guh DP, Zhang W, Bansback N, Amarsi Z, Birmingham CL, Anis AH. The incidence of co-morbidities related to obesity and overweight: a systematic review and meta-analysis. BMC Public Health. 2009;9(1):88.

4. Renehan AG, Tyson M, Egger M, Heller RF, Zwahlen M. Body-mass index and incidence of cancer: a systematic review and meta-analysis of prospective observational studies. Lancet. 2008;371(9612):569-78.

5. Van Gaal LF, Mertens IL, Ballaux D. What is the relationship between risk factor reduction and degree of weight loss? European Heart J Supplements. 2005;7(suppl_L):L21-L6.

6. Vidal J. Updated review on the benefits of weight loss. Int J Obes Relat Metab Disord. 2002;26(S4):S25-8.

7. Wing RR, Lang W, Wadden TA, Safford M, Knowler WC, Bertoni AG, et al. Benefits of modest weight loss in improving cardiovascular risk factors in 
overweight and obese individuals with type 2 diabetes. Diabetes Care. 2011; 34(7):1481-6.

8. Wing RR, Hill JO. Successful weight loss maintenance. Annu Rev Nutr. 2001; 21(1):323-41.

9. Health UDo, Services H. Aim for a healthy weight. Washington: DHHS; 2005. p. 1-38.

10. Jensen MD, Ryan DH, Apovian CM, Ard JD, Comuzzie AG, Donato KA, et al. 2013 AHA/ACC/TOS guideline for the management of overweight and obesity in adults: a report of the American College of Cardiology/American Heart Association task force on practice guidelines and the Obesity Society. J Am College Cardiol. 2014;63(25 part B):2985-3023.

11. QuickStats: age-adjusted percentage of adults aged $\geq 20$ years who tried to lose weight during the past 12 months, by sex - National Health and Nutrition Examination Survey, 2007-2008 to 2015-2016. 2018.

12. Wing RR, Phelan SJTAjocn. Long-term weight loss maintenance- 2005;82(1): 222S-225S.

13. Kraschnewski J, Boan J, Esposito J, Sherwood N, Lehman EB, Kephart DK, et al. Long-term weight loss maintenance in the United States 2010;34(11):1644.

14. Syngal S, Coakley EH, Willett WC, Byers T, Williamson DF, Colditz GA. Longterm weight patterns and risk for cholecystectomy in women. Ann Intern Med. 1999;130(6):471-7.

15. Burke LE, Steenkiste A, Music E, Styn MA. A descriptive study of past experiences with weight-loss treatment. J Am Diet Assoc. 2008;108(4):640-7.

16. Lissner L, Odell PM, D'Agostino RB, Stokes J 3rd, Kreger BE, Belanger AJ, et al. Variability of body weight and health outcomes in the Framingham population. N Engl J Med. 1991;324(26):1839-44.

17. Reynolds MW, Fredman L, Langenberg P, Magaziner J. Weight, weight change, mortality in a random sample of older community-dwelling women. J Am Geriatr Soc. 1999;47(12):1409-14.

18. Arnold AM, Newman AB, Cushman M, Ding J, Kritchevsky S. Body weight dynamics and their association with physical function and mortality in older adults: the Cardiovascular Health Study. J Gerontol Series A: Biomed Sci Med Sci. 2009;65(1):63-70.

19. Nam GE, Cho KH, Han K, Han B, Cho SJ, Roh YK, et al. Impact of body mass index and body weight variabilities on mortality: a nationwide cohort study. Int J Obes (Lond). 2018:1:412-23.

20. Rzehak P, Meisinger C, Woelke G, Brasche S, Strube G, Heinrich J. Weight change, weight cycling and mortality in the ERFORT Male Cohort Study. Eur J Epidemiol. 2007;22(10):665-73.

21. French SA, Folsom AR, Jeffery RW, Zheng W, Mink PJ, Baxter JE. Weight variability and incident disease in older women: the lowa Women's Health Study. Int J Obes Relat Metab Disord. 1997;21(3):217-23.

22. Madigan CD, Pavey T, Daley AJ, Jolly K, Brown WJ. Is weight cycling associated with adverse health outcomes? A cohort study. Preventive medicine. 2018;108:47-52.

23. Bacon L, Aphramor L. Weight science: evaluating the evidence for a paradigm shift. Nutr J. 2011;10(1):9.

24. Mackie GM, Samocha-Bonet D, Tam CS. Does weight cycling promote obesity and metabolic risk factors? Obes Res Clin Pract. 2017;11(2):131-9.

25. Mehta T, Smith DL Jr, Muhammad J, Casazza K. Impact of weight cycling on risk of morbidity and mortality. Obes Rev. 2014;15(11):870-81.

26. Wannamethee SG, Shaper AG, Lennon L. Reasons for intentional weight loss, unintentional weight loss, and mortality in older men. Arch Intern Med. 2005;165(9):1035-40.

27. Schatzkin A, Subar AF, Thompson FE, Harlan LC, Tangrea J, Hollenbeck AR et al. Design and serendipity in establishing a large cohort with wide dietary intake distributions : the National Institutes of Health-American Association of Retired Persons Diet and Health Study. Am J Epidemiol. 2001; 154(12):1119-25.

28. Hermansen SW, Leitzmann MF, Schatzkin A. The impact on National Death Index ascertainment of limiting submissions to Social Security Administration Death Master File matches in epidemiologic studies of mortality. Am J Epidemiol. 2009;169(7):901-8.

29. Atkinson RL, Dietz WH, Foreyt JP, et al. Weight cycling. JAMA. 1994;272(15): 1196-202.

30. Rubin, Donald B. Multiple imputation for nonresponse in surveys. Vol. 81 New York: John Wiley \& Sons, 2004.

31. Smith DL Jr, Yang Y, Nagy TR, Patki A, Vasselli JR, Zhang Y, et al. Weight cycling increases longevity compared with sustained obesity in mice. Obesity. 2018;26(11):1733-9.
32. Sutton EF, Beyl R, Early KS, Cefalu WT, Ravussin E, Peterson CM. Early timerestricted feeding improves insulin sensitivity, blood pressure, and oxidative stress even without weight loss in men with prediabetes. Cell Metabolism. 2018:27(6):1212-21 E3.

33. Longo VD, Panda S. Fasting, circadian rhythms, and time-restricted feeding in healthy lifespan. Cell Metab. 2016;23(6):1048-59.

34. Mattson MP, Allison DB, Fontana L, Harvie M, Longo VD, Malaisse WJ, et al. Meal frequency and timing in health and disease. Proc Natl Acad Sci. 2014; 111(47):16647-53.

35. Anton SD, Moehl K, Donahoo WT, Marosi K, Lee SA, Mainous AG III, et al. Flipping the metabolic switch: understanding and applying the health benefits of fasting. Obesity. 2018;26(2):254-68.

36. Field $A E$, Malspeis $\mathrm{S}$, Willett WC. Weight cycling and mortality among middle-aged or older women. Arch Intern Med. 2009;169(9):881-6.

37. Stevens VL, Jacobs EJ, Sun J, Patel AV, McCullough ML, Teras LR, et al. Weight cycling and mortality in a large prospective US study. Am J Epidemiol. 2012;175(8):785-92.

38. Sarlio-Lähteenkorva S, Rissanen A, Kaprio JJljoo. A descriptive study of weight loss maintenance: 6 and 15 year follow-up of initially overweight adults. 2000;24(1):116.

39. Ferraro ZM, Patterson S, Chaput J-PJCMIE, Diabetes. Unhealthy weight control practices: culprits and clinical recommendations. 2015:8:CMED. S23060.

\section{Publisher's Note}

Springer Nature remains neutral with regard to jurisdictional claims in published maps and institutional affiliations.
Ready to submit your research? Choose BMC and benefit from:

- fast, convenient online submission

- thorough peer review by experienced researchers in your field

- rapid publication on acceptance

- support for research data, including large and complex data types

- gold Open Access which fosters wider collaboration and increased citations

- maximum visibility for your research: over $100 \mathrm{M}$ website views per year

At BMC, research is always in progress.

Learn more biomedcentral.com/submissions 